\title{
Osteochondroma of the talus presenting with tarsal tunnel syndrome - A case report
}

\author{
Pranav Kothiyal ${ }^{1, *}$, Puneet Gupta ${ }^{2}$, Kunal Vij $^{3}$, Akhilesh Singh Kushwaha ${ }^{4}$ \\ ${ }^{1}$ Senior Resident, ${ }^{2}$ Professor \& HOD, ${ }^{3}$ Assistant Professor, ${ }^{4}$ Junior Resident, Dept. of Orthopaedics, Shri Guru Ram Rai Institute \\ of Medical \& Health Sciences, Dehradun, Uttarakhand, India
}

*Corresponding Author:

Email: kothiyalpranav@gmail.com

\begin{abstract}
Osteochondromas are common benign tumours affecting the long bones of the body. Often they are asymptomatic, associated with growing bone and their discovery is incidental. Osteochondromas in the talus are quite rare and their plresence as a cause for tarsal tunnel syndrome is rarer still. Here we report a case of a 10 year old female patient having a osteochondroma of the talus that was presenting with the symptoms of tarsal tunnel syndrome along with tibio-talar arthritic changes. Excision of the bony mass was done which relieved the compressive symptoms and improved the range of motion at the joint. The postop followup has been uneventful at one year now
\end{abstract}

Keywords: Talus, Exostosis, Osteochondroma, Tarsal tunnel syndrome.

\section{Introduction}

One of the most common benign osteocartilaginous neoplasms is the osteochondroma. Out of all the bony tumours osteochondromas constitute about $50 \%$. It commonly involves the long tubular bones like proximal tibial metaphysics, distal femoral metaphysis and proximal humerus occurring in the form of a sessile or pedunculated bony outgrowth covered with a cartilaginous cap. Small tubular bone involvement in hand and foot is less common and flat bone involvement like pelvis and vertebrae is rarer still. An osteochondroma of the talus is very rare. Here we report the case of a talar osteochondroma that presented with symptoms of post traumatic tarsal tunnel syndrome.

\section{Case Report}

A 10 year old female presented to us in the out patient department complaining of pain in her right ankle and foot region. On detailed history taking the patient described that she had developed swelling and pain insidiously in her right ankle about two and a half years ago that was initially mild and was perceived only while walking or running. About two years ago the patient had an episode of trauma, a few months following which she noticed a diffuse swelling around the ankle which was associated with mild pain on walking. Over time the intensity of pain gradually increased along with the size of swelling. Since two months the patient had been complaining of sudden shooting pain in the foot with tingling and numbness along the lateral aspect of the right foot. On examination a diffuse hard swelling was palpated around the anteromedial aspect of right ankle. The swelling was nontender with ill-defined margins. The range of motion at the ankle joint was slightly restricted with 25 degrees of dorsifexion and 20 degrees of plantar flexion. There was partial sensory loss over the dorsum of foot with tingling on percussion over medial and lateral border and along the first ray.

Routine investigations of the patient were done and they were found to be within normal limits. The AP and LAT radiographs of the ankle showed deformity of talar head and beak with a osteocartiilaginous outgrowth from the talar head and neck. A CT scan was done which showed pseudoarticulation between distal tibial epiphysis and the deformed talus. A bony outgrowth measuring $1.5 \mathrm{~cm}$ by $2 \mathrm{~cm}$ by $1.5 \mathrm{~cm}$. was seen arising from the medial aspect of the talus just below the medial malleolus. Flattening and irregularity of the tibio-talar articular surface with cystic changes was also seen.

The patient was managed by open excision of the osteochondroma. Since the swelling was mainly arising from the medial aspect of the talus it was decided to use the anteromedial approach to the ankle joint. A $5 \mathrm{~cm}$ long incision was given over the anteromedial aspect of the ankle joint starting just below and anterior to the medial malleolus extending proximally. Intraoperatively the growth was seen to surround the peroneal and tibial tendons and was exerting pressure over the tarsal tunnel. Degenerative changes were seen in the talar head and the distal tibial articulating surface with cystic changes. The bony mass was excised enbloc after freeing it from the surrounding tendons and the compression over the tarsal tunnel was relieved. Bony fragments were seen lying loose in the joints space and in the surrounding soft tissues which were removed and the final picture was confirmed under fluoroscopy.

Postoperatively the patient was started on physiotherapy of the ankle and was allowed partial weight bearing with walker support on the second postop day and when the intensity of pain decreased by one week she was allowed full weight bearing. The stitches were removed on the 10th postop day. The complains of sharp shooting pain in the foot and 
numbness disappeared after the tumour removal. The patient's range of motion at the ankle improved and the postop followup of the patient has been uneventful since then.

The histopathological examination by haematoxylin and eosin staining revealed bony architecture covered by a hyaline cartilage cap.Regularly arranged cartilage cells in between bony spicules were visualised.

\section{Discussion}

The talus is an unusual location for a bony tumour like an osteochondroma to occur but nevertheless is should be inculded in the differential diagnosis of swellings present around the ankle region. A talar osteochondroma was first reported in 1984 by Fuselier et al. ${ }^{1}$ In 1987, Chioros et al. ${ }^{2}$ reported an osteochondroma from the talus in a adult male. Another osteochondroma was reported by Erler et $\mathrm{al}^{3}$ located on the dorsum of the talus.

Osteochondromas are the most common primary benign bone tumours. ${ }^{4}$ These can be solitary or occur in groups, can be sessile or pedunculated and are composed of cortical and medullary bone, they have a hyaline cartilage cap on the top of the outgrowth. The most common location of these lesions are are in the metaphyseal region of long bones. ${ }^{5}$ Rarely they can arise from atypical locations like flat bones or bones of the foot and even less commonly from the talus. ${ }^{6,7}$

Osteochondromas usually are asymptomatic and generally present as an isolated swelling without any concurrent symptoms. However osteochondroma of the talus can present with pain in the ankle or foot, as a painless ankle mass, decreased range of ankle motion etc as in our patient who had a ankle swelling with restricted range of ankle movements and weight bearing pain.

The cause of pain in a talar osteochondroma could be due to compression of surrounding soft tissue and nerves or from irritation of pain sensitive structures in the vicinity. In our patient the bony outgrowth was impinging on the contents of the tarsal tunnel that is the tibial nerve, posterior tibial artery and tendons of tibias posterior, and the tendons of flexor digitorum longus and flexor hallucis longus causing numbness in the foot and pain radiation to great toe.

Osteochondroma can be easily visualised by $\mathrm{X}$ rays and their extent and morphology can be better delineated by CT scans. ${ }^{8,9}$ X-rays of our patient showed a sessile lesion with regular margins arising from the medial talar aspect with degenerative changes of tibiotalar joint, flattening of talar head and loose bodies in the joint and surrounding soft tissues.

For a symptomatic osteochondroma the treatment is usually to wait and watch. If it is painless, non progressing and cosmetically acceptable then the lesion can be left as such. In case of a symptomatic talar osteochondroma with the patient having complaints like our case did surgical resection is the best treatment. Extraperiosteal complete resection was advocated by Boya et $\mathrm{al}^{10}$ with removal of the cartilage cap to prevent recurrence. In our case the ankle was approached via the anteromedial approach and the bony out growth was excised along with the cartilage cap, the smaller bony protuberances were chiselled off and the loose bodies both intra as well as extraarticular were removed. The prognosis in most of the cases that have been reported in the literature has been good. The followup has been uneventful with relief of symptoms as was seen in our patient who had no fresh complaints till now that is one year after the surgery. There seldom have been any reports of malignant degeneration.

\section{Conclusion}

Osteochondromas in the talus are quite uncommon and those presenting with neurological symptoms in the form of tarsal tunnel syndrome are rarer still. Mostly the benign bony lesion are asymptomatic so they merit no active intervention however in case of a talar osteochondroma presenting with pain, joint morbidity and compression syndromes surgical resection is the best modality of treatment with excellent postop recovery and negligible chances of recurrence.

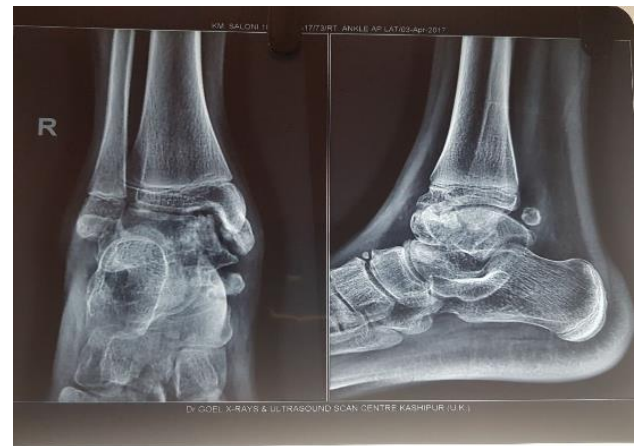

Fig. 1: Preop Xray showing irregular bony growth from medial talar surface 


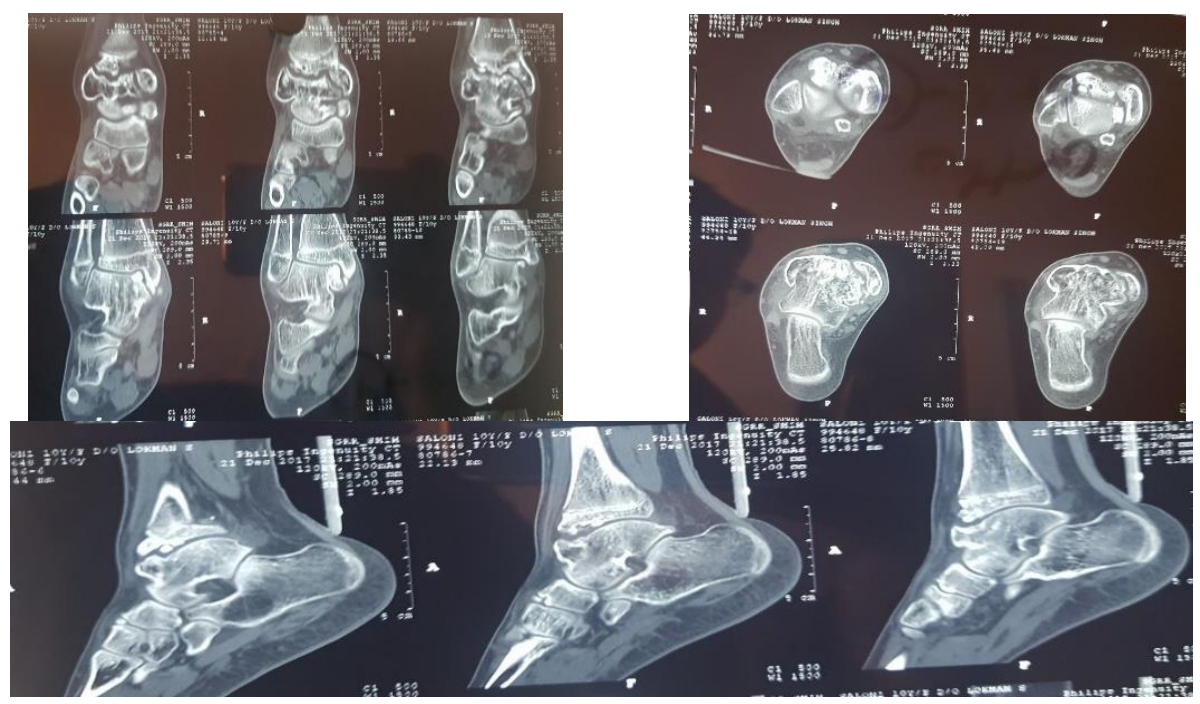

Fig. 2: Coronal and saggital CT scan sections showing osteocartilaginous mass arising from talar surface with deformity of tibio-talar articular surface and loose bodies
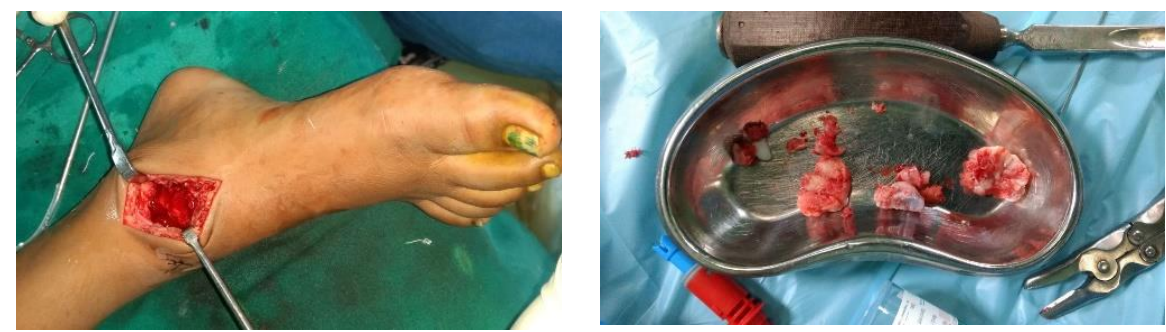

Fig. 3: Histopathological picture of the excised mass showing clusters of active chondrocytes with mature primary trabecular bone

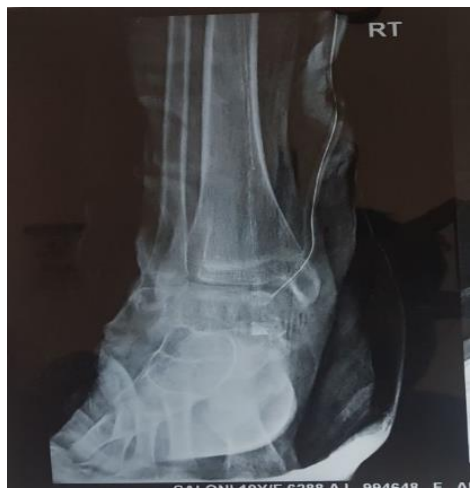

Fig. 4: Postop xray showing the restored normal ankle mortise with the absent bony mass and loose bodies

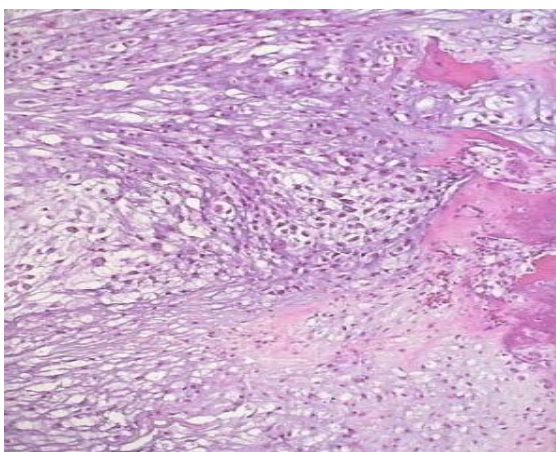

Fig. 5

References

1. Fuselier CO, Binning T, Kushner D, Kirchwehm WW, Rice JR, Hetherington V, et al. Solitary osteochondroma of the foot: an in-depth study with case reports. J Foot Surg. 1984;23(1):3-24. [PubMed]

2. Chioros PG, Frankel SL, Sidlow CJ. Unusual osteochondroma of the foot and ankle. J Foot Surg. 1986;26(5):407-11. [PubMed]

3. Erler K, Oguz E, Komurcu M, Atesalp S, Basbozkurt M. Ankle swelling in a 6-year-old boy with unusual presentation: report of a rare case. J Foot Ankle Surg. 2003;42(4):235-9. [PubMed

4. Saglik Y, Altay M, Unal VS, Basarir K, Yildiz Y. Manifestations and management of osteochondromas: a retrospective analysis of 382 patients. Acta Orthop Belg 2006;72:748-55. 
5. Kawai A, Mitani S, Okuda K, Aoki K, Inoue H. Ankle tumor in a 5-year-old boy. Clin Orthop Relat Res 2003;406:308-316.

6. Joshi D, Kumar N, Singh D, Lai Y, Singh AK.

Osteochondroma of the talus in a male adolescent. J Am Podiatr Med Assoc 2005;95:494-496.

7. Keser S, Bayar A. Osteochondroma of the talar neck: a rare cause of callosity the foot dorsum. J Am Podiatr Med Assoc 2005;95:295-297.

8. Joshi D, Kumar N, Singh D, Lai Y, Singh AK.

Osteochondroma of the talus in a male adolescent. $\mathrm{J}$ Am Podiatr Med Assoc 2005;95:494-496.

9. Keser S, Bayar A. Osteochondroma of the talar neck: a rare cause of callosity the foot dorsum. J Am Podiatr Med Assoc 2005;95:295-297.

10. Boya H, Ozcan O, Tokyol C. Osteochondroma of the talus: an unusual location. Acta Orthop Traumatol Turc. 2014;48(2):236-9. [PubMed] 